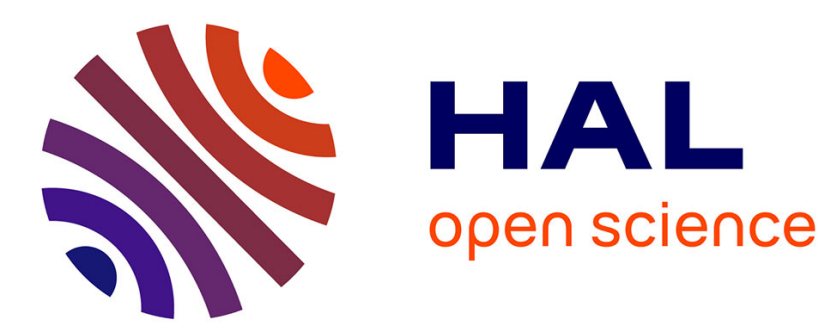

\title{
NEW EXPERIMENTAL RESULTS ON THE HADROPRODUCTION AND PHOTOPRODUCTION OF CHARMED PARTICLES
}

\author{
C. Fisher
}

\section{To cite this version: \\ C. Fisher. NEW EXPERIMENTAL RESULTS ON THE HADROPRODUCTION AND PHOTO- PRODUCTION OF CHARMED PARTICLES. Journal de Physique Colloques, 1982, 43 (C3), pp.C3- 146-C3-151. 10.1051/jphyscol:1982333 . jpa-00221886}

\section{HAL Id: jpa-00221886 \\ https://hal.science/jpa-00221886}

Submitted on 1 Jan 1982

HAL is a multi-disciplinary open access archive for the deposit and dissemination of scientific research documents, whether they are published or not. The documents may come from teaching and research institutions in France or abroad, or from public or private research centers.
L'archive ouverte pluridisciplinaire $\mathbf{H A L}$, est destinée au dépôt et à la diffusion de documents scientifiques de niveau recherche, publiés ou non, émanant des établissements d'enseignement et de recherche français ou étrangers, des laboratoires publics ou privés. 
JOURNAL DE PHYSIQUE

Colzoque C3, suppiément au $n^{\circ} 12$, Tome 43, décembre 1982

page $\mathrm{C} 3-146$

\title{
NEW EXPERIMENTAL RESULTS ON THE HADROPRODUCTION AND PHOTOPRODUCTION OF CHARMED PARTICLES
}

\author{
C.M. Fisher
}

Rutherford Appleton Laboratory, Chilton, Dideot, Oxfordshire, OX11 OQX, U.K.

New data has been submitted to the conference on the production of charmed particles by hadrons and photons. I will briefly review this data, concentrating specifically on the LEBC-EHS hadroproduction experiment (NA16 at CERN).

Previous data from indirect techniques used to study charm hadroproduction, suffer from the fact that the derived cross sections are model dependent and the model parameters can only be inferred from the observed distributions via detailed Monte Carlo calculations. The LEBC-EHS experiment represents a substantially new approach. In this experiment the high resolution hydrogen bubble chamber LEBC has been used to directly observe the charmed particle decay vertices associated with $360 \mathrm{GeV}$ $\pi^{-}$and $360 \mathrm{GeV}$ proton interactions. Production is in hydrogen and therefore there is no "A" dependence correction, and the individual charmed particles are identified by kinematic fits using the spectrometer information. Even when the primary production is very complex the observation of both production and decay:vertices removes the usual combinatorial problems of a pure counter experiment. No attempt is made to select charm using a complex fast trigger; all events are recorded and the charm candidates found from careful scanning and measuring of the high resolution pictures. Events are selected at the scanning stage using conditions based on transverse distances related to the decay kinematics and particle lifetimes but independent of the production $x_{F}$ and $P_{\perp}$. The spectrometer acceptance is high and independent of $x_{F}$ for all $x_{F}>0$. The data is therefore essentially unbiased for the study of production in the forward direction; event weights are small and depend only on decay properties. The defect of NAI6 is the absence of particle identification and as a consequence kinematic ambiguities can exist between $D, F$ and $A^{+}$interpretations for charged charm decays. This problem has been treated in the following way: 1) on $1 \mathrm{y}$ wel1 constrained fits are used ( $3 \mathrm{C}$ or $2 \mathrm{C}$ ); 2) In the case of residual ambiguities a Cabibbo allowed solution is preferred over a Cabibbo supressed solution and a Cabibbo allowed D-solution is preferred over an $F$ or $\Lambda_{c}^{+}$solution.

Evidence for leading plus central production in $\pi^{-} \mathrm{P} \rightarrow \mathrm{D}+\mathrm{X}$

In the NAl6 experiment 29 examples of D-meson production from incident $360 \mathrm{GeV}$ $\pi^{-}$are reported; $10 \mathrm{D}^{-}, 2 \mathrm{D}^{+}, 10 \mathrm{D}^{\circ}, 7 \overline{\mathrm{D}}^{\circ}$. The $\mathrm{D}^{-}(\overline{\mathrm{c}} \mathrm{d})$ and $\mathrm{D}^{\circ}(\mathrm{c} \overline{\mathrm{u}})$ are potentially leading states from the incident $\pi^{-}(\bar{u} d)$ whilst the $D^{+}(c \bar{d})$ and $\bar{D} \circ(c \bar{u})$ are not. $\bar{D}^{\circ}$ however can be fed from a leading $D^{*}=\bar{D}^{\circ} \pi$. Thus the data immediately shows evidence for leading states. In fig. 1 the $x_{F}$ distribution, for $x_{F}>0$, is shown for the potentially leading states ( $1 \mathrm{a}$ ) and the non-leading states ( $1 \mathrm{~b}$ ) separately. The $\mathrm{D}^{-}$and $\mathrm{D}^{\circ}$ distribution extends to high $\mathrm{x}_{\mathrm{F}}$ whilst the $\mathrm{D}^{+}$and $\overline{\mathrm{D}}^{\mathrm{o}}$ are all centrally produced i.e. $\mathrm{x}_{\mathrm{F}}<0.2$. Fig. 1c) shows an integral plot for all states. The lines correspond to $\mathrm{n}=5$ and $\mathrm{n}=1$ for a differential distribution of the form $(1-\mathrm{x})^{\mathrm{n}}$.

The study of prompt muon production in the Fermilab experiment E595 (278 $\mathrm{GeV} \pi^{-} \mathrm{Fe}$ ) also shows evidence for both leading and central production of $D$ mesons. The $\mu^{+}$spectrum implies $\mathrm{D}^{+}, \mathrm{D}^{\circ}$ production like $(1-\mathrm{x})^{3}$ and the $\mu^{-}$spectrum implies $\mathrm{D}^{-}, \mathrm{D}^{\circ}$ like $(1-\mathrm{x})$. Unfortunately these distributions mix leading and non-leading states. For the $\mu^{+}$ distribution the larger leptonic branching ratio for $\mathrm{D}^{+}$is compensated by the larger production (indicated from NA16) of $\mathrm{D}^{\circ}$. The exponent 3 therefore could reasonably arise from a mixture of 1 and 5 . The $\mu^{-}$distribution however derives mainly from the 
$\mathrm{D}^{-}$distribution since the $\mathrm{D}^{-}$has the higher leptonic branching ratio and is strongly produced. Moreover the $\overline{\mathrm{D}}^{\mathrm{O}}$ has also a leading component coming from $\mathrm{D}^{*-}$ decay. Thus the (1-x) ${ }^{1}$ distribution from the $\mu^{-}$spectrum can be taken as evidence for leading production in $\pi^{-} \mathrm{p}$.

Finally the ACCMOR collaboration (NA11) at the SPS observe $\mathrm{D}^{* \pm}$ produced in $175 / 200 \mathrm{GeV} \pi^{-}$Be interactions with a single electron trigger. The distribution is best fjtted by $(1-x)$. Unfortunately $D^{*-}$ (potentially leading) are not separated from $\mathrm{D}^{+}$(non-leading), however, the NAl6 data would indicate that most of the events must be $\mathrm{D}^{*-}$ which would again indicate leading production.

Fig. 2 shows the $\mathrm{p}_{\perp}^{2}$ distribution for the D-mesons observed in NA16. No distinction is observed between the leading and non-leading states; this could however reflect poor statistics. In summary therefore we have evidence for both leading production of the form

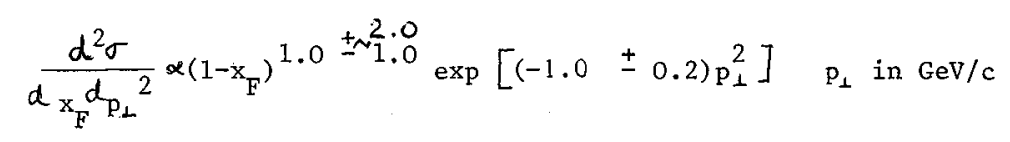

and central production of the form

$$
\frac{d^{2} \sigma}{d_{x_{F}} d_{p_{\perp}}^{2}} \propto\left(1-x_{F}\right)^{6 \pm \sim 2.0} \exp \left[(-1.0 \pm 0.2) p_{\perp}^{2}\right]
$$

in $\pi^{-} \mathrm{p} \rightarrow \mathrm{D}+\mathrm{X}$ at $360 \mathrm{GeV} / \mathrm{C}(\sqrt{\mathrm{s}}=26.8)$. The NA16 data indicates that leading $\mathrm{D}$ states represent about one third of all D's produced.
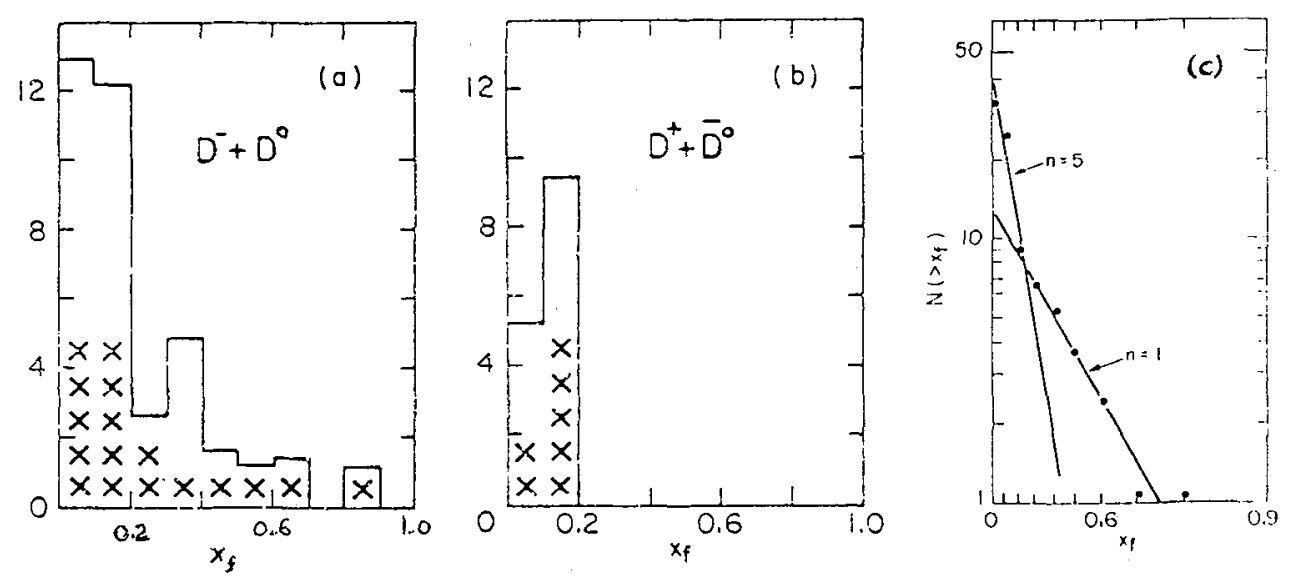

Fig. $1 \times$ distribution for (a) possible leading states $\left(D^{-}+D^{\circ}\right)$; (b) non-leading st tates $\left(\mathrm{D}^{+}\right.$and $\overline{\mathrm{D}}^{\circ}$ ) and $(\mathrm{c})$ the integral distribution for all states in $360 \mathrm{GeV}$ $\pi^{-} \mathrm{p}$ interactions.

Fig. 3 shows the correlations in centre of mass rapidity between reconstructed $\bar{D} \bar{D}$ pairs observed in the NA16 data. A strong correlation exists with $\left\langle\Delta y^{*}\right\rangle=0.49$ for reconstructed pairs. Taking account of the single reconstructed decays in which the second charmed particle is observed in the bubble chamber but is outside of the spectrometer acceptance (i.e. -ve $y^{*}$ ) it is estimated that the mean rapidity (gap between all pairs is $\sim 1.0$. This is consistent with the estimate of Phillips $\left.{ }^{(}\right)_{\text {for }}$ the parton processes $\mathrm{q} \overline{\mathrm{q}} \rightarrow \mathrm{c} \overline{\mathrm{c}}$ and $\mathrm{gg} \rightarrow \mathrm{c} \overline{\mathrm{c}}$ with $\mathrm{m}_{\mathrm{c}}=1.2-1.5 \mathrm{GeV}$. 


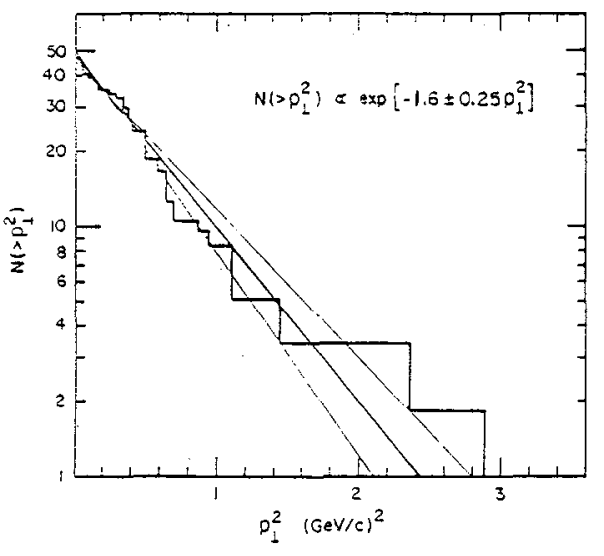

Figure 2

D-meson production in $\mathrm{p}-\mathrm{p}$ collisions at $\sqrt{\mathrm{s}}=26.8$

In $p p \rightarrow D+x$ it is difficult to detect leading particle effects, if they exist, because the only truly, leading particle that can occur is the $\Lambda_{c}^{+}$which takes two out of the 3 valence quarks of the proton. We will discuss the $\Lambda_{c}^{+}$data later. Leading $\mathrm{D}^{-}$of $\overline{\mathrm{D}}^{\circ}$ are expected (see Halzens talk) to behave like $(1-x)^{3}$. Central production with no valence quarks will contribute $\mathrm{D}^{\circ}, \mathrm{D}^{+}, \mathrm{F}^{+}$with a $(1-\mathrm{x})^{5}$ distribution . The $30 \mathrm{D}$-mesons reconstructed in the NA16 data are equally distributed between the $\mathrm{D}$-meson types $\left(7 \mathrm{D}^{-}, 9 \mathrm{D}^{+}, 7 \mathrm{D}\right.$ o and $\left.7 \bar{D}^{\circ}\right)$ i.e. no evidence for any differencebetween $\mathrm{D}^{+} \mathrm{D}^{-}$or $\mathrm{D}^{\circ} \mathrm{D}^{\circ}$ production. No differences are seen between the $x_{F}$ distributions for possible leading D states, i.e. $\mathrm{D}^{-} \overline{\mathrm{D}}^{\circ}$, and non-leading, $\mathrm{D}^{+} \mathrm{D}^{\mathrm{O}}$, states. Fig. 4 shows the integral

$\left(1-x_{F}\right)$ distribution for all states, the slope parameter being $3 \pm 1$ for the differential distribution. The integral $\mathrm{p}_{\perp}^{2}$ distribution has exponent $-1.0_{-0.3}^{+0.2}$ so that the distribution is described by:

$$
\frac{d{ }^{2}}{d x_{F} d p_{i}} \alpha\left(1-x_{F}\right)^{3}-1 \quad \exp \left[(-1.0 \pm: 3) p_{L}{ }^{2}\right]
$$

A mixture of $\left(1-x_{F}\right)^{5}$ and $\left(1-x_{F}\right)^{3}$ cannot be ruled out.

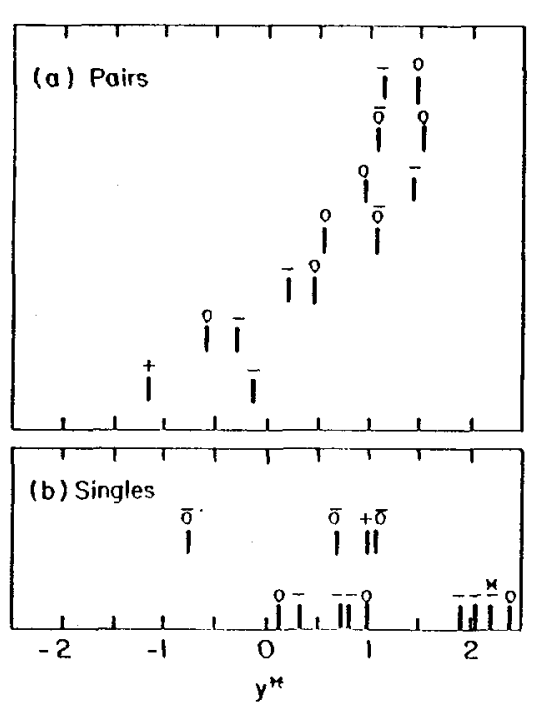

Fig. 3 Rapidity correlations observed between Charm Pairs in the $360 \mathrm{GeV} \pi$ experiment.

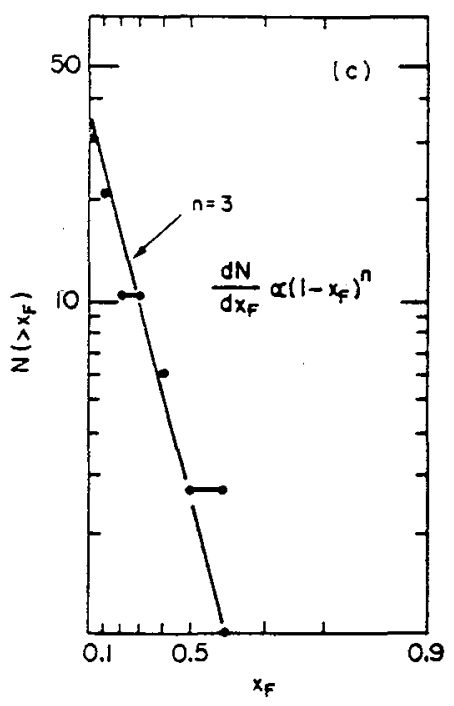

Fig. 4 Integral distribution in $(1-x)$ for $D$-mesons produced in $360 \mathrm{GeV} \mathrm{p}-\mathrm{p}$. 
The E595 experiment using $350 \mathrm{GeV}$ protons into iron find similar results. The ratio $\bar{\mu} / \mu^{+}=1.0 \pm 0.19$ indicating an equal population of $\vec{c}$ and $c$ states at production in agreement with NA16. The data is we11 described by $\operatorname{Ed}^{3} \sigma / \mathrm{dx}_{\mathrm{F}} \sim(1-\mathrm{x})^{6}$ whilst $(1-x)^{3}$ is not a good fit.

Thus the data on $D$-meson production in $\mathrm{p}-\mathrm{p}$ at $\sqrt{\mathrm{s}}=26.8$ shows no obvious evidence for leading quark effects however the statistics are not yet able to sort out a mixture of leading $(1-x)^{3}$ and central (1-x) 5 production.

An interesting feature of the NA16 data is the low numbers of reconstructed pairs in the proton data ( 6 out of $30 \mathrm{D}$ 's appear as pairs compared with 14 out of 29 in the $\bar{\pi}_{p}$ data). This could be due to the other charmed particle in the pair not being a D-meson and being undetected or because the pair of D-mesons has a large rapidity gap so that the other member of the pair is in the backward hemisphere and hence outside of the spectrometer acceptance. The latter explanation is preferred and indicates a large rapidity gap.

$\Lambda_{c}^{+}$and $\mathrm{F}^{ \pm}$production

Direct observation of $\Lambda^{+}$in the bubble chamber data is difficult because (a) the $\Lambda^{+}$appear to be short lived $\left(\tau \sim 2 \times 10^{-13}\right.$ secs) and is therefore at the limit of visibility in the NA16 experiment (not in its successor NA27), (b) kinematic ambiguities exist between the $\Lambda^{+}, \mathrm{F}^{+}$and $\mathrm{D}^{+}$decays which cannot be fully resolved without particle identification ( $\mathrm{c}$ resent in NA27 but not NA16) and (c) the only hadronic (reconstructable) decay mode with known branching ratio is $\wedge_{c}^{+} K_{p}^{-} \pi^{+}$with a small branching ratio $2.2 \pm 1.0 \%$. Nevertheless five events are best interpreted as $\Lambda^{+}$in the NAI6 p-p data compared with one event in the $\pi^{-}$p data. Similar difficulties exist for $\mathrm{F}^{+}$and again the observed ratio is 5 in $\mathrm{p}-\mathrm{p}$ compared with 1 in $\pi^{-}$.

The $\mathrm{x}_{\mathrm{F}}$ distribution for all constructed decays in the NA16 p-p experiment is shown in $\mathrm{fig}^{\mathrm{F}}$. 5. The identified $\Lambda_{\text {c }}^{+}$events have relatively high $\mathrm{x}_{\mathrm{F}}$ and could be part of a (1-x) distribution. The $F$ events (apart from one which could be a misidentified D) are by comparison more central. Because of the uncertainties in event identification and the sensitivity vs $x$ at very short lifetimes it would be dangerous to read too much into these few events. The final story awaits the NA27 proton data.

Indirect evidence exists both in NA16 and E595 against any large_production of $\Lambda_{c}^{+}$in association with $\bar{D}^{\circ}$. Thus $\Lambda_{c}^{+}$production will be accompanied by $\bar{D}^{\circ}$ if it is the result of a simple fragmentation of the incident proton and we might therefore expect to see an excess of $\bar{c}$ states amongst the $\mathrm{D}$-mesons $i . e$. more $\bar{D}$ than $\mathrm{D}$. In the NA16 data however we have $12 \mathrm{c}$ quark states $\left(\mathrm{D}^{+}\right.$or $\mathrm{D}^{\circ}$ ) and $16^{\mathrm{c}}$ states_( $\mathrm{D}^{-}$or $\overline{\mathrm{D}}^{\mathrm{O}}$ ) i.e. a small excess of c states. Similarly the E595 experiment finds $\mu^{-} / \mu^{+}=1.0 \pm 0.19$ i.e. equal $c$ and $\bar{c}$.

Thus from these two experiments there is no evidence for $\Lambda^{+} \bar{D}^{\circ} / \mathrm{D}^{-}$production at a level greater than a few microbarns at $\sqrt{\mathrm{s}}=27$. The upper limit is difficult to quantify but is about $20 \mu \mathrm{b}$. The ACCMOR collaboration reported a $\Lambda_{\mathrm{c}}^{+}$signal associated with $\sum c \stackrel{++}{\rightarrow} \Lambda^{+} \pi^{+}$in their initial analysis of $200 \mathrm{GeV} \mathrm{p}$-Be events, however, with about a factor 5 increase in statistics they no longer see the signal and quote an upper limit on the cross-section $\sigma\left(\mathrm{p}+\mathrm{p} \rightarrow \hat{\lambda}_{\mathrm{c}}^{+}+\mathrm{X}\right) \leqslant 24 \pm 16 \mu \mathrm{b}$ at $90 \% \mathrm{C}$.L.

Two experiments however report results indicating large $\Lambda_{c}^{+}$cross sections. The BIS-2 collaboration working in a neutron beam at about $60 \mathrm{GeV}$ at Serpukhov report 10 and 46 peaks in the $K^{\circ} p \rightarrow K^{\circ} \mu^{\top} \pi^{+}$and $\Lambda^{\circ} \pi^{+} \pi^{+} \pi^{-}$final states at $2275 \pm 6 \mathrm{MeV} / \mathrm{c}^{2}$. A linear A dependence yields $44 \mp 16,+22 \mu \mathrm{b}$ for the cross section and the production distributions are fitted by $\left(1-\mathrm{x}_{\mathrm{F}}\right)^{1.5 \pm 0.4}$ exp $\left(-2.7 \pm 0.7 \mathrm{p}_{\perp}\right)$. An emulsion search for charged decays to three charged particles without reconstruction has been made by the Bombay-Chandigarh-Delhi-Tammin Collaboration. Because of the short distances involved a signal of 8 events is interpreted as due to $\Lambda^{+}$production (assuming $\left.\tau \sim 10^{-13} \mathrm{secs}\right)$ and leads to a high cross section $92 \pm 35 \mu \mathrm{b} /$ nucleon at $400 \mathrm{GeV} / \mathrm{c}$. Other interpretations however are possible and the absence of any kinematic reconstruction means that this should probably be interpreted as an upper limit to the $\Lambda_{\mathrm{c}}^{+}$crosssection. 


\section{Hadroproduction cross sections}

Using the decays with known branching ratios the NA16 experiment yields:

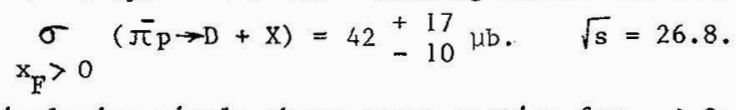

This is an inclusive single charm cross section for $x_{F}>0$; the pair cross section would equal this value if $\underset{x_{F}>0}{\sigma}=\underset{x_{F}<0}{\sigma}$.

The cross section derived from the E595 experiment is model dependent. Using the best fit to the $(1-x)$ distributions described above they find:

$$
\begin{gathered}
\sigma(\bar{\pi} p \rightarrow D+x)=8.2 \pm 0.9 \mu b \text { and } \sigma\left(\bar{\pi}_{p} \rightarrow \bar{D}+x\right)=9.5 \pm 0.7 \mu b \\
x_{F}>0 \\
x_{F}>0
\end{gathered}
$$

This is again an inclusive single charm cross section for $x_{F}>0$ and the pair cross-section would equal this value if $\underset{\mathrm{x}_{\mathrm{F}}>0}{\sigma}=\frac{\sigma_{\mathrm{F}}}{\mathrm{x}_{\mathrm{F}}}$.

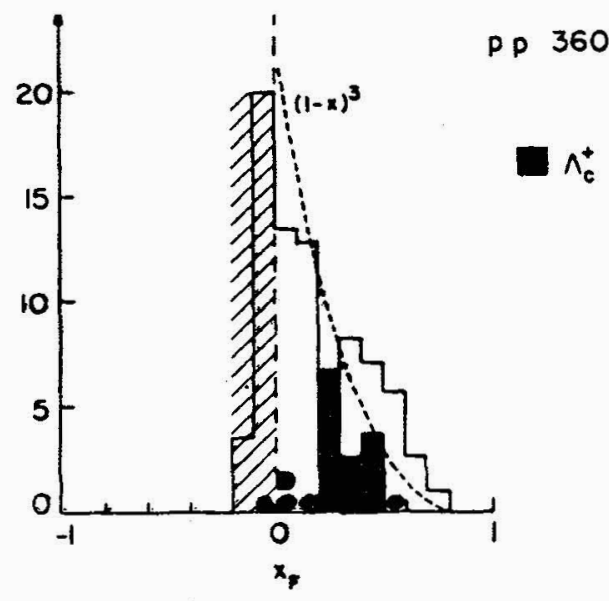

Fig. 5 Distribution in $x_{F}$ for all charmed particles in $360 \mathrm{GeV} \mathrm{p}-\mathrm{p}$ interactions. $\Lambda_{c}^{+}$in squares, $\mathrm{F}^{ \pm}$ in solid circles (NA16 data).

Similarly the two experiments give independent measurements of the charm pair production by protons at $\sqrt{\mathrm{s}}=26.5$. Symmetry about $\mathrm{x}_{\mathrm{F}}=0$ allows the determination of the total pair cross section:

$$
\begin{aligned}
& \text { NA16 } \sigma(\mathrm{pp} \rightarrow \mathrm{D}+\text { charm }+\mathrm{x}) \simeq 38 \pm \sim 16 \mu \mathrm{b} \\
& \mathrm{E} 595 \sigma(\mathrm{pp} \rightarrow \mathrm{c} \overline{\mathrm{c}}+\mathrm{x}) \quad \simeq 24 \pm 2 \pm \mu \mathrm{b}{ }^{*} \text {. }
\end{aligned}
$$

Note that the E595 result is model dependent; depending on the exponent $n$ of $(1-x)^{n}$ at production, the mean semi-leptonic branching ratio for the mix of states produced, and the " $A$ " dependence used in scaling from production in iron. Values used are $n=6$, $B R=8 \%$, and $A \frac{1}{273}$ If $n=3$ is assumed the $E 595$ cross section reduces by a factor 2 whilst if an $\mathrm{A}^{2 / 3}$ dependence on atomic number were used the resulting cross-section would increase by a factor 3.8 . Thus to make the results compatible we can either have $n$ large i.e. mostly central production and $A^{1}$ (preferred by the data) or $n$ small $\sim 3$ i.e. diffractive production, and $A^{2 / 3}$. It could well be that central and diffractive production have different A dependences and that some mix is involved as in the $\pi$ case.

The ACCMOR collaboration also give a model dependent cross.section, however using a model basically in agreement with the NA16 and E595 data but with the additional assumption that $D^{*} / D=1$ at production. They find:

$$
\text { NA11 } \left.\sigma_{(\bar{\tau} \mathrm{p} \rightarrow \mathrm{DD}}+\mathrm{x}\right)=(34 \pm 8) \mu \mathrm{b} \pm 24 \mu \mathrm{b} \text {, for } \sqrt{\mathrm{s}}=20 \text { and }
$$




$$
\frac{\sigma(\sqrt{s}=15.5)}{\sigma(\sqrt{s}=20)}=0.41 \pm 0.15
$$

New photoproduction data

The SLAC Hybrid Facility Photon Collaboration have given a Charm photoproduction cross section near threshold. They report:

$\sigma(\gamma \mathrm{p} \rightarrow$ Charm $)=57_{-25}^{+30}$ nanobarns at $20 \mathrm{GeV}$.

This experiment has many of the positive features described above for NA16. The SLAC $40^{\prime \prime}$ bubble chamber equipped with a high resolution optics scannel ( $\left.55 \mu \mathrm{m}\right)$ is used to directly detect the decay vertices of the charmed particles. The events are clean because production is near threshold and hence the vertices are not overlaid by other particles from production. The monochromatic photon beam is produced by back scattering a laser on the $20 \mathrm{GeV}$ SLAC electron beam. The detection efficiency and hence cross section estimate however, depends on a number of factors viz.: the type of particle produced i.e. $\mathrm{D}^{+} \mathrm{D}^{-}, \mathrm{D}^{\circ} \mathrm{D}^{\mathrm{O}}, \overline{\mathrm{D}}^{\circ} \Lambda^{+}$etc., the dynamics of production, and the decay characteristics of the individual particles. The low beam energy results in relatively large decay angles and hence poor acceptance downstream. These questions are resolved by Monte Carlo techniques and the resulting uncertainties are reflected in the quoted errors. Nevertheless the result gives a cross-section close to threshold and is extremely valuable in estimating the resulting threshold s-dependence.

The CERN-Omega Photoproduction collaboration have repeated a search for F-meson production in $\gamma-p$ interactions between 50 and $70 \mathrm{GeV} / \mathrm{c}$. Data was taken using the tagged photon beam with the trigger requiring a forward charged multiplicity between 2 and 5 together with at least one photon greater than $2 \mathrm{GeV}$. The $\gamma \gamma$ mass distribution shows a very significant $\eta$-peak after photons that can be associated to make $\pi^{\circ}$ mesons have been removed. An F-signal is observed in $\eta \pi^{t}\left(17 \pm 6\right.$ events), $\eta \pi^{t} \pi(14 \pm 9)$

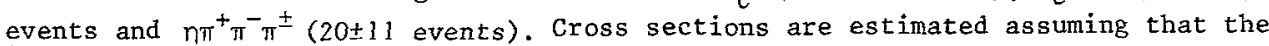
$\mathrm{F}$-mesons are produced in pairs in a photon-gluon fusion model and carry the same forward momentum as the cc pair. A cut requiring the observed $F^{ \pm}$to have momentum greater than $20 \mathrm{GeV}$ is applied; equivalent to observation only above $x_{F}=0.25$. The resulting model dependent estimates for the inclusive cross section to produce on $\mathrm{F}^{+} \mathrm{F}^{-}$pair are given in the table.

\section{$\underline{\text { Table } 1}$}

$\begin{array}{llcr}\text { Decay Channel } & \text { No events } & \text { Efficiency, \% } & \sigma . \mathrm{B}(\mathrm{nb}) \\ \mathrm{n \pi}^{ \pm} & 17 \pm 6 & 1.83 & 38 \pm 14 \\ \mathrm{n \pi}^{ \pm} \pi^{\circ} & 14 \pm 9 & 0.85 & 66 \pm 42 \\ \mathrm{n \pi}^{+} \pi^{-} \pi^{ \pm} & 20 \pm 11 & 0.87 & 93 \pm 52 \\ \mathrm{n \pi}^{+} \pi^{-} \pi^{\circ} \pi^{+} & <10 & 0.19 & <216\end{array}$

\section{Acknowledgements}

I would like to thank my colleagues in the NA16 Collaboration, Drs Halzen, Phillips, Bodek, Ringland, Moffeit and Galbraith for very valuable discussions before and during the conference. 